\title{
Diseño de un instrumento que identifique bajo la percepción del empleado el desarrollo del proceso administrativo de una PyME
}

\author{
Bertha Nayalek Escareño Dojaquez ${ }^{1}$, Karla Emilia Cervantes Collado ${ }^{2}$ y Gloria Muñoz Del Real ${ }^{3}$ \\ ${ }^{1}$ Universidad Autónoma de Baja California, Facultad de Ciencias Administrativas, nayalek.escareno@uabc.edu.mx, \\ Calzada de los presidentes y Eje central, Río Nuevo, 21120 Mexicali, B.C., (+52) 6862300296 \\ ${ }^{2}$ Universidad Autónoma de Baja California, Facultad de Ciencias Administrativas, kkarla@ uabc.edu.mx, Calzada \\ de los presidentes y Eje central, Río Nuevo, 21120 Mexicali, B.C, (+52) 6861570699 \\ ${ }^{3}$ Universidad Autónoma de Baja California, Facultad de Ciencias Administrativas, gloria.munoz@uabc.edu.mx, \\ Calzada de los presidentes y Eje central, Río Nuevo, 21120 Mexicali, B.C., (+52) 6861199350
}

Información del artículo revisado por pares

Fecha de aceptación: junio-2021

Fecha de publicación en línea: diciembre-2021

DOI: https://doi.org/10.29105/vtga7.1-112

\section{Resumen}

Actualmente las Pequeñas y Medianas Empresas se han interesado más por obtener ganancias que por la implementación de un adecuado proceso administrativo. Por su parte, los empleados van creando percepciones sobre este proceso en la empresa donde laboran, el cual se consideran un tema importante a estudiar internamente. Una percepción es subjetiva y depende de cada individuo, pero puede ser un factor que impacte directamente en la organización. Para conocer cómo miran los trabajadores al proceso administrativo, se tiene como objetivo en este trabajo, el diseño de un instrumento que permita medir la percepción del empleado sobre el mismo. El análisis realizado se basó en sus cuatro etapas, tales como la planeación, organización, dirección y control. El instrumento desarrollado se validó aplicando a una población muestra, lo que permitió la integración de item’s que logran medir la percepción del empleado sobre la ejecución del proceso administrativo.

Palabras clave: Proceso administrativo, planeación, organización, dirección, control, PYME

Códigos JEL: D01, M10, M14

\section{Resume}

Currently, Small and Medium Enterprises have been more interested in obtaining profits than in the implementation of an adequate administrative process. For their part, employees are creating perceptions about this process in the company where they work, which is considered an important issue to study internally. A perception is subjective and depends on each individual, but it can be a factor that directly impacts the organization. In order to know how the workers look at the administrative process, the objective of this work is the design of an instrument that allows to measure the employee's perception of it. The analysis carried out was based on its four stages, such as planning, organization, direction and control. The instrument developed was validated by applying it to a sample 
population, which allowed the integration of items that manage to measure the employee's perception of the execution of the administrative process.

Keywords: Administrative process, planning, organization, direction, control, SME

JEL Codes: D01, M10, M14

\section{1-. INTRODUCCIÓN}

Planteamiento del problema

Uno de los problemas en las Pequeñas y Grandes Empresas (PYMES) es el poco interés sobre el proceso administrativo, puesto que lo primero que busca una empresa de este tamaño es recurso financiero, poniéndolo como número uno en su lista de prioridades y no poniendo atención en los demás aspectos de su empresa según el análisis en PYMES de Bohórquez y Cendales (2014).

El presente estudio consiste en la validación de un instrumento que logre medir bajo la percepción de los empleados, si el proceso administrativo aplicado a una PYME es correctamente implementado. El interés del desarrollo de este instrumento surge del análisis del caso de estudio realizado a una PYME que inició operaciones en el año 2000. Esta empresa se dedica a la atención de problemas presentados en HVAC; es decir por sus siglas en inglés Heating, Ventilation and Air Conditioning. En un plazo de dos años la empresa tuvo un crecimiento del $150 \%$ en sus ventas, lo que provocó que de 15 colaboradores fuera necesario aumentar a 70 en los últimos dos años. Actualmente esta empresa presenta problemas específicos en controles y procedimientos en el proceso administrativo, derivados del crecimiento obtenido.

Apoyar este tipo de empresa, es necesario para el crecimiento del país, así como lo menciona Benavides (2019). Las cifras del Directorio Estadístico Nacional de Unidades Económicas del 2018, muestra que existen en nuestro país un total de 5 millones 78 mil 737 unidades económicas, de las cuales 4,756,690 tienen un tamaño de establecimiento de 10 o menos personas, lo que significa que representan el 93 por ciento. Es por ello que el proceso administrativo busca obtener una organización fuerte que pueda enfrentar cualquier obstáculo y pueda llegar a alcanzar sus objetivos.

Pregunta de Investigación

¿La validación de un instrumento de medición podrá identificar bajo la percepción del empleado cuál de las etapas del proceso administrativo como planeación, organización, dirección y control se encuentran correctamente implementadas?

Hipótesis

La validación de un instrumento de medición que pueda identificar la percepción de los empleados sobre las etapas del proceso administrativo ayudará a conocer cuales se encuentran correctamente implementadas.

Objetivo

Validar el instrumento de medición que pueda identificar la percepción del empleado sobre las dimensiones del proceso administrativo como planeación, organización, dirección, control para conocer cuales se encuentran correctamente implementadas.

\section{2-. MARCO TEÓRICO}


En la presente sección se habla de las definiciones expuestos por diferentes autores, para así, entender la situación que se investiga en este trabajo.

\section{Proceso Administrativo}

Para este trabajo es importante hablar del proceso administrativo puesto que es la base para profundizar en cada etapa, Palencia (2003) menciona que es el sistema que contiene diferentes funciones, en él se encuentra la planeación, organización, dirección y control. Cada uno de ellos utilizan los recursos materiales y humanos que tiene la empresa para lograr sus objetivos. Estas etapas se complementan una con la otra, creando un desarrollo de actividades más eficiente. El tamaño de una empresa no importa al momento de facilitar el proceso hacia el cumplimento de los objetivos de la empresa aun así es un proceso que contiene una carga de responsabilidad alta puesto que planea y regula todas las operaciones para que se pueda llegar al propósito por el cual fue creada.

El proceso administrativo para Dubrin (2000) consta de una seria de pasos para que se llegue a un producto o servicio excelente y así poder tener una utilidad. El proceso administrativo se debe de manejar de forma adecuada para beneficio de la empresa. Lo que se toma en cuenta son los recursos financieros así como el dinero de la empresa, los recursos humanos donde se refiere al personal, los recursos físicos como la infraestructura de las oficinas y recursos tecnológicos que son las computadoras o impresoras.

Para las pequeñas y medianas empresas (PyMES), el mejorar su administración, es lo único que les confiere competir con otras empresas, en otras palabras, lograr optimizar la coordinación de los recursos que posee, tanto los físicos como humanos, además, de tener un análisis del entorno externo, puesto que todo esto es superada por sus grandes competidoras (Reyes, 2004).

Para este trabajo se optó por dividir al proceso administrativo en cuatro etapas como se menciona en esta sección, puesto que son con las que más se identifica la empresa.

\section{Planeación}

Para Hernández y Palafox (2012), nos dice que en la planeación se debe de tomar en cuenta información del pasado y su entorno, esto para poder organizar, dirigir y medir el desempeño de la empresa y la de sus integrantes, a través de controles que pueda cotejar lo que se planeó contra lo que se realizó. Para tener más claro lo que abarca la planeación, se identificaron ciertas preguntan que responden a su definición como: ¿Qué debe hacerse? Haciendo énfasis en la actividad que se debe de realizar. ¿Por qué debe hacerse? Esta pregunta se dirige a la razón de lo que se planea. ¿Cuánto y cuándo debe hacerse? Se habla de la cantidad y específicamente del tiempo cuando se debe de realizar. ¿Quién debe hacerlo? Identifica el área, departamento y persona que realizara lo planeado. ¿Cómo debe hacerse? Se dirige a las acciones que se van a realizar en específico.

Robbins y Coulter (1996) nos dan una serie de pasos de la etapa de planeación, tanto como su práctica como su evaluación. El primer paso se refiere a identificar la misión de la empresa, además de los objetivos y sus estrategias; esto ayudara a que la gerencia identifique el campo del producto o servicio con más detalle. El segundo se refiere a realizar un análisis al entorno externo, puesto que el entorno delimita las opciones disponibles para que la gerencia pueda tomar una decisión. Sobre el tercer paso se habla sobre el análisis de los recursos de la empresa, dado que cada una de las entidades tiene cierto limites en sus recursos y habilidades. La formulación y la implementación de estrategias seria nuestro cuarto paso, y este es necesario para que la gerencia desarrolle y evalué las opciones que estén más acorde al área a aplicar. Por 
último, tenemos el quinto paso donde se habla de la evaluación de resultados, dejando en claro que se basa en evaluar lo que se logró en la aplicación de la estrategia, en este paso se observa la efectividad y el grado de cumplimiento de los objetivos, logrando conocer si es necesario realizar algún diseño o ajuste.

\section{Organización}

La organización para Rodríguez (2011) la divide por etapas las diferentes actividades que se tienen que efectuar para organizar o reorganizar a una empresa.

La primera de ellas es la división del trabajo, que se define en separar y delimitar las actividades. $\mathrm{Al}$ dividir las actividades se logra tener mejores resultados, puesto que se tiene más precisión al realizarlas y se logra la eficiencia con un mínimo esfuerzo. No es ser eficiente cuando alguien intenta hacer de todo. Se necesita tener diferencias y especializaciones al realizar algo, sin importar el tamaño que tenga la empresa. Así mismo, a cada empleado no se le integran todas las actividades, muy probablemente puedan desviarse y provocar a que la empresa no cumpla con ella misma ni con el cliente. (Hitt et al, 2006).

Se dice sobre la Jerarquización como por orden de rango, funciones o importancia. (Martínez, 2017). Los diferentes niveles en una empresa tienden a ser el conjunto de dependencias que se encuentran unidos por su grado de autoridad y responsabilidad, sin importar las actividades que realicen. También en la Jerarquización se lleva a cabo la definición de la estructura organizacional, mediante áreas de autoridad y comunicación para que se puedan relacionar entre sí.

La Departamentalización especifica que es la división y el agrupamiento de las funciones y actividades específicas, con base en su similitud. Galvez, Hernandez y Molina (2016), hace referencia sobre la departamentalización como la subdivisión de las tareas y la asignación de éstas a grupos especializados de la empresa, así como en la creación de normas para el desempeño de esas funciones.

De acuerdo con Stoner, et al (1996), otro aspecto importante en la Organización es coordinar lo que se encuentra dentro del proceso. La coordinación une las funciones de los departamentos independientes con el fin de alcanzar los objetivos organizacionales con eficacia. (Valda, 2012)

\section{Dirección}

El concepto de Dirección para Stoner (1996) la define como la etapa que se asegura o garantiza que una estructura se desarrolle hacia el objetivo definido y cumpliendo con la función diseñada.

También Newman (1996) menciona que dentro de la dirección es donde se aplica la autoridad y se transfieren instrucciones, esto teniendo en cuenta la posible respuesta de las personas a las que van dirigidas. La autoridad se debe de usar para que se realicen las actividades asignadas a cada equipo de trabajo, el utilizarlo incorrectamente podría traer consecuencias fuertes para la organización.

Dentro de la dirección se encuentra la motivación, que de acuerdo con Santrock (2002), es el conjunto de razones por las que las personas se comportan de las formas en que lo hacen. El comportamiento motivado es vigoroso, dirigido y sostenido. Madrigal (2009) alude a que es una situación complicada de abordar y hablar para los empleados. Aun así, cuando se llega la hora de cuestionar a una persona sobre como motivar, esta responde a un ambiente relacionado a los reconocimientos de carácter económico. Hoy en día, los estímulos económicos alcanzan a motivar al empleado, pero solo por un tiempo limitado, estos se pueden identificar fácilmente, pero en el momento en el que la empresa no otorgue ese beneficio, los trabajadores pueden reaccionar de manera inmediata, puesto que entienden que es obligación de la empresa 
y desencadena una serie de acontecimientos. Walter (2003) nos menciona que los procesos que intervienen en la motivación son: Capacitación, remuneraciones, condiciones de trabajo, clima organizacional, relaciones humanas, políticas de contratación, seguridad, liderazgo y sistemas de recompensa.

El liderazgo, siendo muy importante para la dirección, Pratt (1997) la define como un procesosituación en el que una persona, en sus cualidades físicas, valores y simpatía o en su capacidad para resolver problemas de la vida cotidiana, encuentra seguidores que actúan bajo su influencia. El liderazgo en una empresa es indispensable, puesto que es la que la lleva en dirección correcta para alcanzar sus objetivos. Así como se dice que el capitán del barco o el director de una orquesta son lo más importante, el papel puede ser fundamental para una organización. El ser líder de una empresa no quiere decir que sea el dueño de esta, cuando se es una grande es probable que no suceda estos casos, al contrario de una pequeña o mediana empresa que tiene a ser la misma persona. (E.B., 2016).

\section{Control}

Al Control, Ponce lo define (2012) como establecer un sistema que permita medir los resultados actuales y los esperados, con el objeto de conocer si se ha obtenido lo que se esperaba, modificar, mejorar o formular planes. Esta etapa es un elemento importante para el proceso administrativo debido a que permite que la empresa se encuentre regulada en términos deseados. Para comprender la importancia del control interno en las empresas lo primero es empezar por entender el propósito. El control tiene como objetivo resguardar los recursos de la empresa o negocio evitando pérdidas por fraude o negligencia, como así también detectar las desviaciones que se presenten en la empresa y que puedan afectar al cumplimiento de los objetivos de la organización. Desarrollar un Control Interno adecuado para cada organización nos permitirá optimizar la utilización de recursos con calidad para alcanzar una adecuada gestión financiera y administrativa, logrando mejores niveles de productividad. (Servin, s.f.)

Para su comprensión, Stoner. J (1996) indicó que los elementos que tiene el Control son: establecer estándares de desempeño puesto que se debe establecer medidas de control, en donde indique el desempeño del personal en cada actividad y si realmente se están alcanzando los resultados esperados. También se deben de medir los resultados con las normas establecidas, debemos controlar si realmente el personal cumple las instrucciones y normas que se ha establecido para cada puesto. Además de tomar decisiones o medidas correctivas cuando se detectan desviaciones al observar si los resultados no son satisfactorios, debemos tomar decisiones para corregirlas lo más pronto posible.

Dentro de control se abarca el monitorio, donde se puede definir como un proceso que se encarga de estar recolectando y analizando datos para utilizar la información y poder darles seguimiento a los avances de una planeación y así poder guiar las decisiones de acuerdo con lo que surja. Este se dirige a los procesos que responden al cómo, cuándo y dónde de las actividades, además, del quién y a cuantas personas afecta. Por lo tanto, el monitoreo comienza al momento de haber iniciado la planeación y continua en todo el tiempo de la implementación. En ocasiones al monitoreo se le puede llamar evaluación formativa. (Organización de las Naciones Unidas, 2010).

\section{3-. MÉTODO}

Para construir y realizar la validación de un instrumento de medición fue necesario un análisis bibliográfico, lo que permitió diseñar la operacionalización de la variable. El instrumento obtenido busca identificar bajo la percepción del empleado, cuál de las etapas del proceso administrativo como; planeación, organización, dirección y control se encuentran correctamente implementadas (ver tabla 1). El instrumento 
indaga sobre la falta de claridad en la descripción de las actividades, en la asignación de actividades, en el liderazgo por departamento, en la retroalimentación y seguimiento de problemas.

A continuación, se determinó que la variable de proceso administrativo consiste en cuatro dimensiones; planeación se compone del conocimiento de actividades, identificación de la planeación y análisis de los recursos; organización se aborda con los indicadores de división del trabajo, jerarquía, departamentalización y coordinación; dirección considera la motivación, capacitaciones, remuneraciones y liderazgo; y por último, control describe las medidas correctivas y monitoreo (ver tabla 1).

Tabla 1 Operacionalización de la variable.

\begin{tabular}{|c|c|c|}
\hline \multirow{2}{*}{ VARIABLE } & DIMENSION & INDICADOR \\
\hline \multirow{3}{*}{$\begin{array}{c}\text { PROCESO } \\
\text { ADMINISTRATIVO }\end{array}$} & PLANEACION & $\begin{array}{c}\text { CONOCIMIENTO DE ACTIVIDADES } \\
\text { IDENTIFICACION DE LA PLANEACION } \\
\text { ANALISIS DE LOS RECURSOS }\end{array}$ \\
\cline { 2 - 3 } & ORGANIZACIÓN & $\begin{array}{c}\text { DIVISION DE TRABAIO } \\
\text { JERARQUIA }\end{array}$ \\
\cline { 2 - 3 } & DEPARTAMENTALIZACION \\
& COORDINACION \\
\cline { 2 - 3 } & DIRECCION & MOTIVACION \\
& CAPACITACIONES \\
& REMUNERACIONES \\
& CIDERAZGO \\
\cline { 2 - 3 } & MENTROL & MEDIDAS CORRECTIVAS \\
& MONITOREO \\
\hline
\end{tabular}

Fuente: Elaboración propia del autor.

El desarrollo de la operacionalización de la variable dio como instrumento de medición un cuestionario que se integra de 35 reactivos con escala tipo Likert que contiene cinco posibles respuestas. Para la validación del instrumento se realizó la prueba piloto a un número específico de sujetos de estudio, sumando en total 13 empleados. El software de análisis fue el IBM SPSS stadistics 20 del cual se obtuvieron las pruebas de alfa de Cronbach, KMO, esfericidad de Barlett y comunalidades.

\section{4-. RESULTADOS}

La primera prueba realizada fue el Alpha de Cronbach, donde según Lee J. Cronbach (1951) los resultados se encuentran en intervalos de 0 a 1 , considerando al 0.70 como bueno; 0.80 favorable; 0.90 excelente. Los resultados obtenidos en las pruebas realizadas al instrumento de evaluación diseñado en este trabajo de investigación fueron las siguientes; Planeación obtuvo 0.818; Organización de 0.848; Dirección 0.845 y Control 0.868 , lo que significa que el instrumento es confiable, debido a que los parámetros de Alpha de Cronbach alcanzaron puntuaciones clasificadas como favorables.

La prueba de Kaiser, Meyer y Olkin mejor conocida por KMO, permite comprobar el grado de relación conjunta entre las variables, los parámetros identificados como válidos para esta prueba deben estar 
entre 0.5 y 1 (Crombet-Grillet et al., 2019). Los resultados que arrojó dicha prueba al instrumento analizado fue de 0.546 en relación con las cuatro dimensiones de la variable del proceso administrativo, es decir, las dimensiones se encuentran relacionadas entre sí.

La prueba de Maurice Stevenson Barlett (1937) muestra que los resultados deben ser menores a 0.005 para determinar la homogeneidad de varianzas, lo que significa que esta prueba permite contrastar la igualdad de la varianza en más de dos poblaciones. El instrumento aplicado obtuvo 0.002 en su prueba de Barlett, es decir que los resultados son homogéneos.

La última prueba realizada al instrumento para su validación fue la prueba de comunalidades obteniendo para planeación 0.846; organización 0.929; dirección 0.781; y control 0.929, esta prueba explica en mayor proporción la varianza según su participación en los factores. Zamora Muñoz et al. (2010) menciona que los resultados deben ser mayor a .04, lo que significa que este instrumento cuenta con los parámetros aceptables de variabilidad de cada dimensión explicada.

En la tabla 2 se concentran los resultados obtenidos en cada una de las pruebas descritas, una vez realizada la extracción de los item's con menor carga factorial.

Tabla 2 Pruebas realizadas.

\begin{tabular}{|c|c|c|c|c|c|c|c|c|}
\hline \multirow{3}{*}{ VARIABLE } & \multirow{3}{*}{ DIMENSION } & \multirow{3}{*}{ INDICADOR } & \multirow{3}{*}{ REACTIVOS } & \multirow{3}{*}{ ITEMS } & \multicolumn{4}{|c|}{ PRUEBAS REALIZADAS } \\
\hline & & & & & $<.7$ & $<.5$ & $.000-.005$ & $<.4$ \\
\hline & & & & & $\begin{array}{l}\text { ALPHA DE } \\
\text { CRONBACH }\end{array}$ & KMO & BARLETT & COMUNALIDADES \\
\hline \multirow{4}{*}{$\begin{array}{c}\text { PROCESO } \\
\text { ADMINISTRATIVO }\end{array}$} & PLANEACION & $\begin{array}{l}\text { CONOCIMIENTO DE ACTIVIDADES } \\
\text { IDENTIFICACION DE LA PLANEACION } \\
\text { ANALISIS DE LOS RECURSOS }\end{array}$ & 01-08 & 8 & 0.818 & \multirow{4}{*}{0.546} & \multirow{4}{*}{0.002} & 0.846 \\
\hline & ORGANIZACIÓN & $\begin{array}{c}\text { DIVISION DE TRABAIO } \\
\text { JERARQUIA } \\
\text { DEPARTAMENTALIZACION } \\
\text { COORDINACION } \\
\end{array}$ & 09-13 $15-18$ & 9 & 0.848 & & & 0.929 \\
\hline & DIRECCION & $\begin{array}{l}\text { MOTIVACION } \\
\text { CAPACITACIONES } \\
\text { REMUNERACIONES } \\
\text { LIDERAZGO } \\
\end{array}$ & $19-27$ & 9 & 0.845 & & & 0.781 \\
\hline & CONTROL & $\begin{array}{l}\text { MEDIDAS CORRECTIVAS } \\
\text { MONITOREO }\end{array}$ & 31-34-35 & 3 & 0.868 & & & 0.929 \\
\hline
\end{tabular}

Fuente: Elaboración propia del autor.

\section{5-. CONCLUSIONES}

El diseño y validación del instrumento planteado en este trabajo, confirma la hipótesis donde se refiere a que se puede identificar la percepción de los empleados sobre el proceso administrativo con las etapas de planeación, organización, dirección y control, en el cual ayudó a realizar los análisis correctos para poder proponer mejoras al proceso. De igual forma, se cumplió con el objetivo al validar el instrumento de medición, puesto que pudo identificar la percepción del empleado como antes se mencionó. Gracias a que, al obtener respuestas satisfactorias en el desarrollo de este artículo, se continuara con la investigación que se encuentra abierta para ser aplicado al total de la población. 
La percepción que tienen los empleados del proceso administrativo impacta en el desarrollo de sus actividades. Se puede decir que es indispensable tener una buena percepción para que la empresa llegue a sus objetivos. Medir la percepción que tienen los empleados puede ayudar a implementar las acciones correctas y encontrar el proceso que mejor se ajuste. El tener un proceso administrativo adecuado se obtiene una variedad de ventajas, entre ellas se puede decir que la dirección de la empresa se dirige a sus objetivos, elimina operaciones que no agregan valor al producto o servicio, facilitan la toma de decisiones o la creación de métodos que ayuden a las diferentes áreas.

La aplicación de las pruebas requirió la eliminación de un reactivo en la dimensión de Organización y cinco reactivos en Control. La ejecución de estos reactivos fue consecuencia de no obtener un resultado favorable en las pruebas de fiabilidad y validación. 


\section{REFERENCIAS}

Crombet, S., Abalos, A. y Zamora, L. (2019). Determinación de los parámetros ambientales de mayor incidencia en las aguas residuales de la comunidad universitaria Antonio Maceo. Revista Cubana de Química, 31(1), 137-153.

Cronbach, L. (1951) Coefficient alpha and the internal structure of test. Psychometrika.

Dubrin, A. (2000). Fundamentos de Administración. Editorial Thomson.

E.B. (25 de abril de 2016) La importancia del liderazgo en el mundo de la empresa. El boletín. https://www.elboletin.com/economia/133362/importancia-liderazgo-mundo-

Gálvez, E., Hernández y Molina, V. (2016). Departamentalización e innovación en las micro, pequeñas y medianas empresas de Colombia. Revista Venezolana de Gerencia, 21. (74)272-287. https://www.redalyc.org/articulo.oa?id=29046685007

Hernández, S. y Palafox, G. (2012). Administración. Teoría, proceso, áreas funcionales y estrategias para la competitividad. Tercera edición. McGraw-Hill.

Hitt, M., Black, S., y Porter, L. (2006). Administración. Pearson

Madrigal, B. (2009). Habilidades Directivas. Mc Graw Hill/ interamericana Editores S.A.

Martínez, F. (2017). La división del trabajo y su influencia en la productividad de las MYPES ubicadas en el distrito de la ciudad nueva de la región de Tacna 2017. [Tesis de licenciatura, Universidad privada de Tacna]. http://repositorio.upt.edu.pe/bitstream/UPT/709/1/Martinez-CaceresFiorella.pdf

Newman, W. y Warren, H. (1996). Administración Dinámica. Editorial Diana.

Organización de las Naciones Unidas. (31 de octubre del 2010). ¿Cuál es el monitoreo y la evaluación?. https://www.endvawnow.org/es/articles/330-cul-es-el-monitoreo-y-la-evaluacin.html

Palencia, R. (2003). Aplicación del proceso administrativo en un sistema de costos para las microempresas de cerámica de Santa Cruz Chinautla. Guatemala. [Tesis de licenciatura].

Ponce, A. (2012). Gestión Empresarial. Interamericana editores S.A. de C.V.

Pratt, H. (1997). Diccionario de Sociología. FCE.

Reyes, A. (2014). Administración de empresas, teoría y práctica. Limusa.

Robbins, S., Stephen, P. y Coulter, M. (1996). Administration. Pearson Educación.

Rodríguez, S. (2011). Fundamentos de Gestión empresarial. Mexicana.

Santrock, J. (2002). Psicología de la educación. Mc Graw-Hill.

Servín, L. (s.f.). ¿Por qué es importante el control interno en las empresas?. Deloitte. https://www2.deloitte.com/py/es/pages/audit/articles/opinion-control-interno-empresas.html

Stoner, J. (1996). Administración. Editorial Prentice Hall Hispanoamérica. 
Valda, J. (2012). Grandes Pymes. https://www.grandespymes.com.ar/2012/11/06/tecnicas-deorganizacion-y-coordinacion-en-la-empresa/

Walter, A. (2003). La motivación en la empresa. https://www.gestiopolis.com/la-motivacion-en-laempresa/

Zamora, S., Monroy, L., y Chávez, C. (2010). Análisis factorial: Una técnica para evaluar la dimensionalidad de las pruebas Cuaderno técnico 6 [Review of Análisis factorial: Una técnica para evaluar la dimensionalidad de las pruebas Cuaderno técnico 6, por A. Saade Jazin]. Centro Nacional de Evaluación para la Educación Superior, A.C. (Ceneval). 


\section{ANEXOS}

\section{Anexo 1}

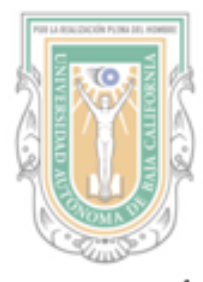

\section{UNIVERSIDAD AUTONOMA DE BAJA CALIFORNIA FACTULDA DE CIENCIAS ADMINISTRATIVAS MAESTRIA EN ADMINISTRACIÓN}

La presente encuesta es parte de una investigación que tiene como objetivo diseñar un plan basado en la cultura organizacional para la mejora del proceso administrativo. La encuesta es confidencial y anónima, por lo cual sea lo más sincero posible.

Por favor lea detenidamente y marque con una $\mathrm{X}$ la respuesta correcta.

Edad:

Departamento:

Género:

Antigüedad en la empresa:

\begin{tabular}{|c|c|c|c|c|c|c|}
\hline No. & Pregunta & $\begin{array}{c}\text { Totalmente } \\
\text { Acuerdo }\end{array}$ & $\begin{array}{c}\text { Miy } \\
\text { Acuerdo }\end{array}$ & \begin{tabular}{c|} 
De \\
Acuerto
\end{tabular} & $\left|\begin{array}{c}\text { En } \\
\text { Desacuerdo }\end{array}\right|$ & $\begin{array}{c}\text { Totalmente } \\
\text { en }\end{array}$ \\
\hline & Planeacion & & & & & \\
\hline 1 & $\begin{array}{l}\text { Considera usted que h empresa es chra en asignar actividades? } \\
\end{array}$ & & & & & \\
\hline 2 & ¿Usted sabe el proposito de sus activida des cuando se has destinan? & & & & & \\
\hline 3 & Conoce cuando debe de realizar la actividad cuando se h señalan? & & & & & \\
\hline 4 & Con frecuencia tiene chro quien debe realizar ciertos babores? & & & & & \\
\hline 5 & $\begin{array}{l}\text { ¿Sabe usted como se deben de realizar las actividades cuando se le } \\
\text { asignan? }\end{array}$ & & & & & \\
\hline 6 & Conoce los objetivos de ha empresa? & & & & & \\
\hline 7 & $\begin{array}{l}\text { ¿Considera usted que ha empresa realiza una correcta planeacion } \\
\text { sobre sus actividades? }\end{array}$ & & & & & \\
\hline 8 & $\begin{array}{l}\text { ¿La empresa le otorga todas has herramientas para usted pueda } \\
\text { realizar su trabajo? }\end{array}$ & & & & & \\
\hline & Organización & & & & & \\
\hline 9 & $\begin{array}{l}\text { ¿Considera usted que el organigrama de su empresa esta bien } \\
\text { estructurado? }\end{array}$ & & & & & \\
\hline 10 & $\begin{array}{l}\text { ¿Usted cree que están bien definidas los niveles jerarquicos su } \\
\text { empresa? }\end{array}$ & & & & & \\
\hline 11 & Para usted serin escencial tener un organigrama bien definido? & & & & & \\
\hline 12 & $\begin{array}{l}\text { ¿Considera que sus actividades haborales están defnidas } \\
\text { correctamente? }\end{array}$ & & & & & \\
\hline 13 & ¿Considera que la division de su trabajo esta a decuada a su puesto? & & & & & \\
\hline 14 & $\begin{array}{l}\text { ¿Usted cree que las actividades de su departamento estan } \\
\text { delimitadas? }\end{array}$ & & & & & \\
\hline 15 & Buscan los mismos objetivos en su departamento? & & & & & \\
\hline 16 & $\begin{array}{l}\text { ¿Tiene una buena coordinacion entre sus compañeros de trabajo } \\
\text { dentro de su departamento? }\end{array}$ & & & & & \\
\hline 17 & $\begin{array}{l}\text { ¿Existe coordinación entre su área de trabajo y los otros } \\
\text { departamentos? }\end{array}$ & & & & & \\
\hline 18 & Se tiene una buena coordinacion entre usted y su jefe directo? & & & & & \\
\hline
\end{tabular}




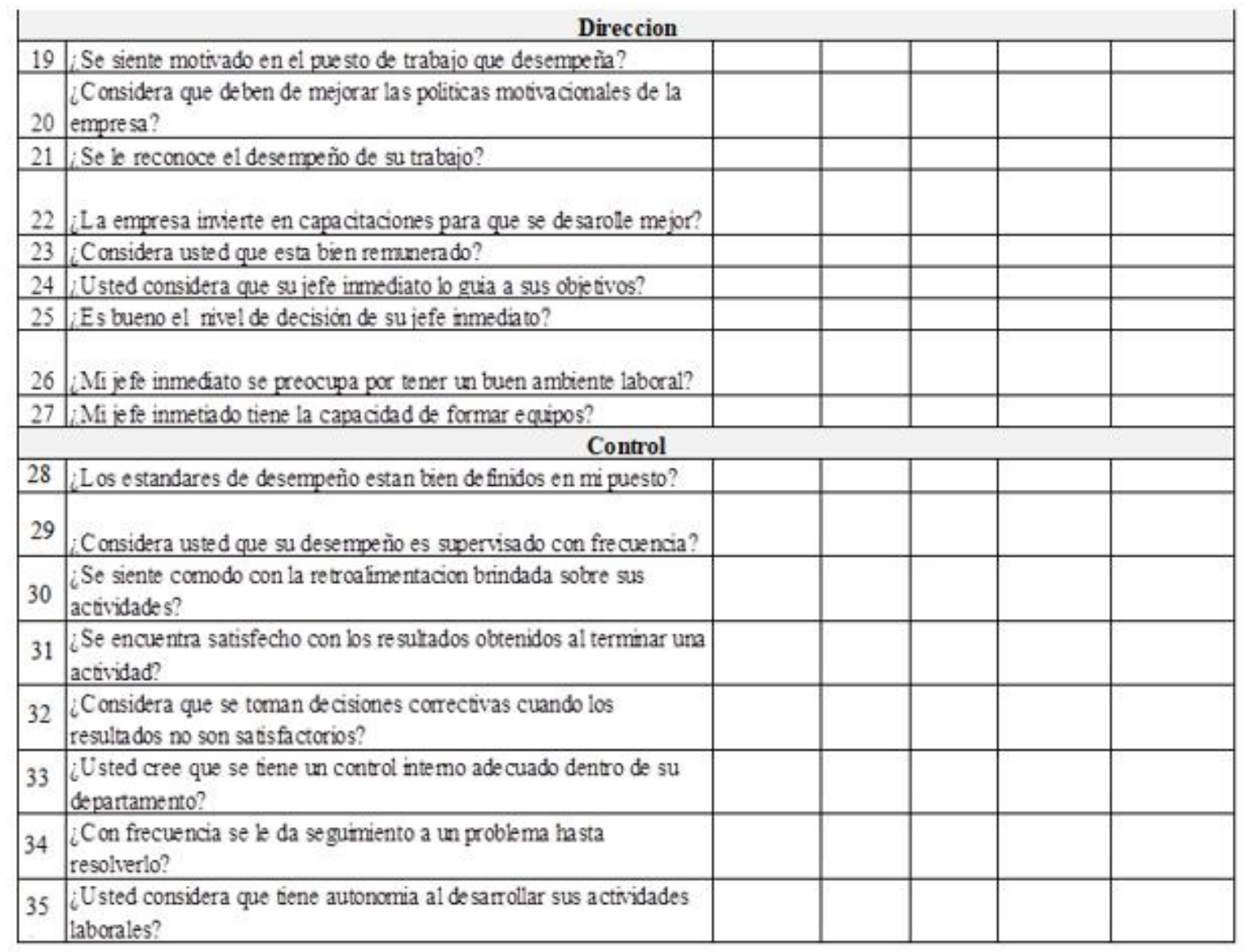

